\section{A minimalist technique for insertion of intrauterine devices}

\author{
Norman David Goldstuck \\ Department of Obstetrics and \\ Gynaecology, Faculty of Medicine and \\ Health sciences, Stellenbosch University \\ and Tygerberg Hospital, Cape Town, \\ South Africa
}

\section{Abstract}

The world's population is approaching 7 billion. As a general rule, the countries with the highest population have the least available healthcare resources, the most notable exception being the United States of America (USA). Most of these countries have an urgent need to reduce their populations. The intrauterine device (IUD) is used by the largest number of contraceptives world-wide and it has a proven record in reducing unwanted pregnancies. Its efficacy rate as a long-acting reversible contraceptive is matched only by subdermal implants which are not as cost effective. Although the rates of pelvic infection are elevated in many countries with low-resource health care systems, we now know that pelvic infection rates are independent of IUD usage. This is therefore no longer a contraindication for using IUDs on a large scale in family planning programs. The technique of IUD insertion as described in most textbooks and journals is unnecessarily complex and based on ritual rather than good clinical evidence. This is particularly interesting in that at a time where we prefer evidence based medicine there are still so many clinical practice sacred cows. This article advocates a simplification of the technique for inserting IUDs. The scientific rationale for simplifying the technique is presented, as well as evidence that it is as safe if not safer than the currently suggested methods, if used for the correct type of IUD acceptors

\section{Introduction}

The instruments which are used for inserting IUDs are historically those used for gynaecological procedures. During these procedures the subject is usually anaesthetised or provided with analgesia. This is not usually the case with IUD insertion, especially in low resource settings. Thus, an Allis forceps ${ }^{1-4}$ may be preferable to using a sharp toothed tenaculum as it prevents the IUD provider from attempting the insertion too forcefully as it will lose grip if more than $6 \mathrm{~N}$ of force are applied while it is attached to the cervix. ${ }^{5}$ It thus acts as a safety-valve to the use of excessive force, and causes less pain than a conventional single toothed tenaculum. The correct time for inserting an IUD is there and then. ${ }^{6}$ There is no time during the menstrual cycle that an IUD cannot be placed in a suitable candidate, and some compelling reasons why insertions at times other than during the menstrual period may be preferable. $^{6}$

\section{Technical Note}

Other than the IUD itself, the minimalist technique requires only 3 pieces of equipment: i) a sterilised or disposable speculum, ii) an Allis forceps (preferably) or a sharp toothed tenaculum, also sterilised and iii) a scissors (preferably long and curved in that it does not come into contact with body tissue at any stage so that it only needs to be surgically clean). Additionally, some disinfectant solution, e.g. povidone-iodine and cotton ball swabs are required. These are shown in Figure 1.

\section{Discussion}

This technique is designed to ensure the fastest, most comfortable, and quickest way to ensure an intrauterine device is placed. It is assumed that at least a perfunctory history has been taken this may take only 2-3 minutes. A detailed pelvic examination for screening for general gynaecological examination is not performed. The assumption is made that it has either recently been completed or that resources are so limited that the necessary testing, e.g. pap smears, bacteriology etc. is not available and only resources for family planning are.

This technique is applicable for interval insertion (insertion at least 8 weeks post-partum or post-abortion only). It should be used with great caution in women who are lactating or have been on injectable contraception for over 12 months since these women are known to be more susceptible to uterine perforation. ${ }^{7}$

The only equipment which is needed is shown in Figure 1. Either a disposable or a sterilised speculum can be used, as well as the remainder of the instruments as previously described. A rapid pelvic examination will exclude pelvic infection, gross uterine or adnexal abnormalities and give an indication of the position of the uterus. The next steps are as follows. First, insert the speculum and view the cervix. The position of the cervix will very often confirm if the uterus is anteverted or retroverted. Second, using the Allis forceps
Correspondence: Norman David Goldstuck, Department of Obstetrics and Gynaecology, Faculty of Medicine and Health sciences, Stellenbosch University and Tygerberg Hospital, Cape Town, Western Cape 7505, South Africa.

Tel: +27.823418200 .

E-mail: nahumzh@yahoo.com

Key words: Intrauterine device; insertion; gynaecology.

Received for publication: 24 January 2015. Accepted for publication: 24 January 2015.

This work is licensed under a Creative Commons Attribution 3.0 License (by-nc 3.0).

(C) Copyright N.D. Goldstuck, 2015

Licensee PAGEPress, Italy

Healthcare in Low-resource Settings 2015; 3:5067 doi:10.4081/hls.2015.5067

hold the back of a cotton-ball swab and dip it into a povidine-iodine disinfectant solution, or equivalent. Swab the cervix. Third, withdraw the marker on the IUD stem (the one that is usually set to uterine length) and insert the IUD until the fundus is felt, in the same way one would do when using a uterine sound. Release the device into the cavity using the mechanism appropriate for the device. Fourth, cut the threads to the appropriate length and remove the speculum.

This technique omits the sounding of the uterus which is considered a quintessential procedure before IUD insertion for which there is no one established piece of evidence. ${ }^{8}$ There are no controlled studies with or without the use of a uterine sound before IUD insertion. Here are some reasons why sounding is not advisable. First, it is possible to perforate the uterus in vitro with a metal sound with 20 $\mathrm{N}$ of force. Second, it is not possible to perforate the uterus in vitro with most types of IUD as they will bow. ${ }^{8}$ The degree of bowing is however unknown for the Mirena ${ }^{\circledR}$. Third, uterine sounding for establishing total uterine axial length is an inherently geometrically useless operation. ${ }^{9}$ It is the attempt to make a one dimensional assessment of a three dimensional organ (the uterus), before inserting a two dimensional product (the IUD). Fourth, a simple understanding of geometry and topology will make obvious that in this situation, uterine sounding is more a ritual than a scientific procedure. Uterine sounding may however be of some limited value prior to insertion of the Gyne Fix IUD, because it is a one dimensional device. Sounding with metal sounds has been shown to be inaccurate. ${ }^{9}$ This inaccuracy may lead to an inaccurate placement of the IUD so that it is not placed at the uterine fundus which makes it more likely that the device will be expelled, or if its position in the uterine cre- 


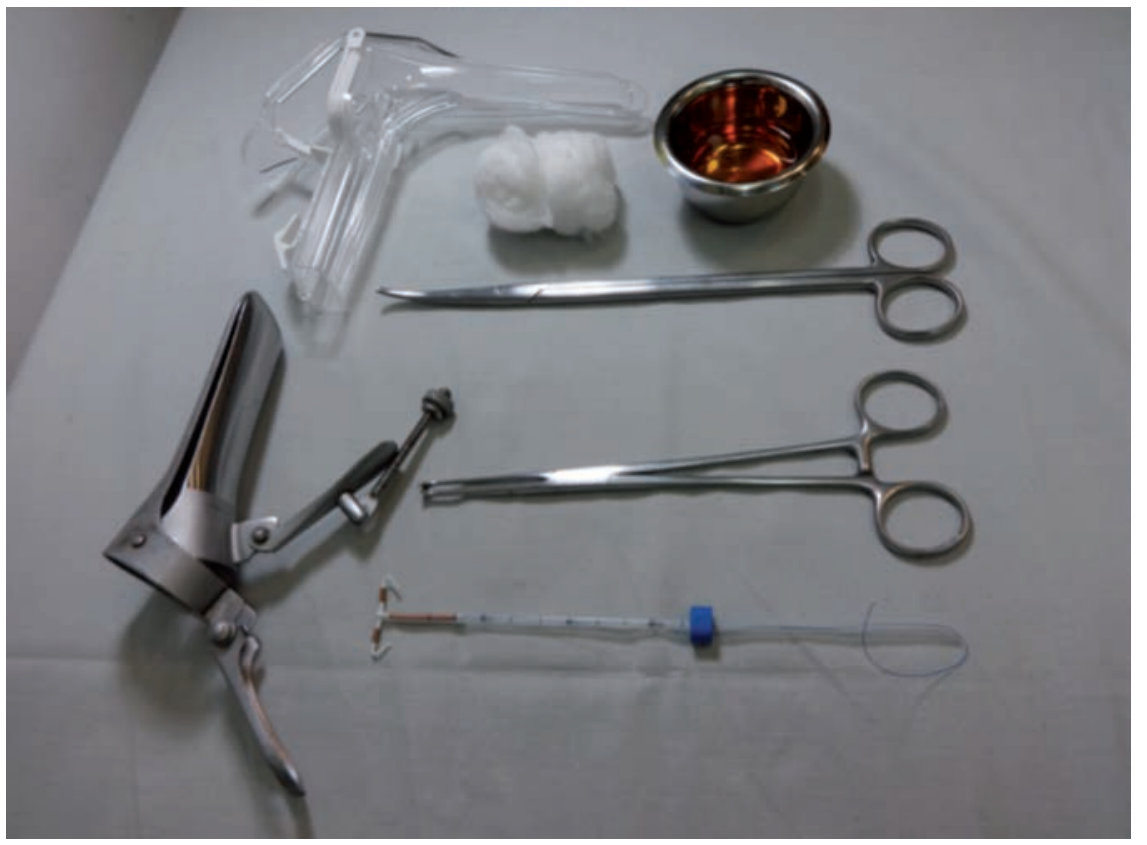

Figure 1. Layout of all the instruments and appliances needed for minimalist intrauterine device insertion.

ates sufficient asymmetrical uterine muscle forces than embedment, partial or complete perforation may be the result. ${ }^{7}$

\section{Conclusions}

This technique can be used by all IUD providers with confidence in women who have had children. While the intrauterine device has been shown to be appropriate for nulliparous women, this technique should only be used by very experienced providers in the nulliparous group. By using this method and inserting IUDs with lifetimes of 10 years and more large numbers of women in low resource countries could receive adequate contraception at minimal cost as it is possible to get the TCu 380A from some manufacturers for as little as $\$ 10$ for bulk purchases. A minimal amount of mainly re-useable instruments has only ongoing costs related to re-processing. There is a minimal cost of consumables. Finally, the ability to perform insertions this way very rapidly produces staff cost savings.

\section{References}

1. Department of Economic and Social Affairs, Population Division of the United Nations. World contraceptive use 2007. Available from: http:/www.un.org/esa/population/publications/contraceptive2007/co ntraceptive2007.htm

2. Winner B, Peipert JF, Zhao Qiuhony Z, et al. Effectiveness of long-acting reversible contraception. New Engl J Med 2012;36:1 998-2007.

3. Farley TM, Rosenberg MJ, Rowe PJ, et al. Intrauterine devices and pelvic inflammatory disease: an international perspective. Lancet 1992;339:785-8.

4. Goldstuck ND. Insertion of intrauterine devices: some technical considerations. Practitioner 1979;223:647-51.

5. Goldstuck ND. Insertion forces with intrauterine devices: implications for uterine perforation. Eur J Obstet Gyn R B 1987;25:315-23.

6. Whiteman MK, Tyler CP, Folger SG, et al. When can a woman have an intrauterine device inserted? A systematic review. Contraception 2013;87:66-73.

7. Goldstuck ND, Wildermeersch D. Role of uterine forces in intrauterine device embedment, perforation and expulsion. Int J Womens Health 2014;6:735-44.

8. Goldstuck ND. 'Bowing' forces with IUD insertions in vitro: reference to difficult IUD insertions. Clinical Rep Fertil 1987;5:173-6.

9. Goldstuck ND. The mark - 7 sound - an accurate determinant of uterine axial length. Contraception 1979;20:359-65. 